\title{
Employee motivation to participate in workplace innovation via in-house crowdsourcing
}

\author{
Kira Palin, Valtteri Kaartemo
}

\begin{abstract}
Crowdsourcing has rapidly gained popularity in business and academia. Research on crowdsourcing has focused mostly on the resources external to an organisation, with less attention being paid to in-house crowdsourcing (IHCS). We believe that IHCS has potential to empower employees to partake in workplace innovation. We argue that employee motivation is one of the most significant factors influencing the success or failure of any IHCS project. Yet, to the knowledge of the authors, employee motivation has not been studied in the context of IHCS thus far, and the present study aims to fill this research gap. We present the case of an IHCS project launched by a large Finnish government-owned company (GOC) that offers diversified logistics services. We identify five factors that influence employees' extrinsic and intrinsic motivation to participate in workplace innovation via IHCS, namely working environment, rewards systems, feedback and processing time, user experience and role of technology, and site marketing and communication. Further, we set several theoretical propositions for empirical testing. Our contribution to workplace innovation literature is two-fold: enhancing the general understanding of IHCS; and studying employee motivation in the IHCS context. We also contribute to the wider innovation management literature by increasing general knowledge about innovativeness of GOCs.
\end{abstract}

Keywords: workplace innovation, employee motivation, in-house crowdsourcing, government-owned companies 


\section{Introduction}

The concept of crowdsourcing, that is, outsourcing an organisational function to an undefined (and generally large) network of people in the form of an open call, has spread among companies over the past decade and is currently facing increasing demand from both the crowd and companies. Coined by Howe (2006), crowdsourcing has rapidly increased its presence in contemporary business, in addition to being studied widely by academics.

The traditional view of crowdsourcing focuses on the resources external to an organisation. Yet, there is another significant form of crowdsourcing used within companies, namely, internal, in-house, or intra-corporate crowdsourcing (Villarroel \& Reis 2010; Stieger et al. 2012; Simula \& Vuori 2012). According to Villarroel and Reis (2010, p. 2) intra-corporate crowdsourcing refers to "the distributed organisational model used by the firm to extend problem-solving to a large and diverse pool of self-selected contributors beyond the formal internal boundaries of a multi-business firm: across business divisions, bridging geographic locations, leveling hierarchical structures." Companies using internal crowdsourcing employ modern technological tools to receive ideas, suggestions, and solutions from their own employees (Stieger et al. 2012). In addition, internal crowdsourcing benefits from the knowledge of a heterogeneous employee pool (Simula \& Ahola 2014). Thus, there is a clear linkage to workplace innovation, which refers to "new and combined interventions in work organisation, human resource management and supportive technologies” (Pot 2011, pp.404-405). Particularly, workplace innovation discusses work organisation, structure \& systems, reflection and innovation, workplace partnership, and 'the fifth element' - the interdependence between these workplace practices (Totterdill 2015).

In the traditional innovation process, management is considered to make decisions on innovations (Kesting \& Ulhøi 2010). It has been thought that the cognitive bias of employees prevents them from understanding the "bigger picture," which is required for the innovation process to be commercially successful. This is thought to be one of the reasons why only a fraction of employee innovation ideas are perceived as relevant (Kesting \& Ulhøi 2010). In these situations, an in-house crowdsourcing (IHCS) platform would be a handy workplace innovation practice to empower employees to challenge established practices and suggest own ideas to the senior management. As Simula and Ahola (2014) point out, in IHCS not all ideas go all the way to the management, but they are filtered through the systems of the IHCS platform. Although IHCS platforms and systems filter employees' ideas through the process, IHCS could be contrasted with intra-organisational knowledge transfer, which has been noted to motivate employees to share their knowledge within an organisation (Aalbers et al. 2013).

The IHCS concept has not been addressed widely within academia (Stieger et al. 2012). However, companies such as Nestlé have already implemented IHCS by creating an internal social network and peer-to-peer knowledge sharing systems that enable all Nestlé workers to share their ideas with their colleagues. Employees can discuss and suggest development ideas to an extent not possible before. In addition to the organisational effects of internal crowdsourcing, Internet technology frees employees from the bounds of working hours or physical workplaces. Moreover, online collaboration allows contributors from diverse backgrounds to participate in the process, which is one of the main success factors of any crowdsourcing process. 
Overall improvement in the extent of reach and abundance of information, as well as the rich diversity of respondents, are aspects that can be counted as the main positive factors of IHCS (Stieger et al. 2012). Yet, these positive results are not exclusive to the most progressive multinationals. To underline this, we present the case of an IHCS project launched by a large Finnish government-owned company (GOC) that offers diversified logistics services.

We argue that one of the most significant factors affecting the success or failure of any IHCS project is the degree of employee motivation. Change in the overall job description or a small increase in the amount of work is likely to result in rejection, resistance, or possibly both. Therefore, in order for a company to secure the success of a desired project, it should be able to motivate its workers in a correct manner. Yet, to the knowledge of the authors, employee motivation has not been studied yet in the context of IHCS, and through the current study, the authors aim to fill this research gap.

The purpose of this study is to identify the factors that motivate employees to engage in workplace innovation via IHCS. Our contribution to workplace innovation literature is two-fold. First, we enhance the general understanding of IHCS, which has remained largely unearthed in the literature. Second, we contribute to the literature on employee motivation and innovation by linking the two ideas in the context of IHCS. More broadly, we contribute to research on the innovativeness of GOCs. Innovation processes in GOCs have been studied to some extent, and the results have been fairly diverse. Research indicates that GOCs are not able to capture the benefits of open innovation (Väätänen et al. 2011). On the other hand, federal agencies in the U.S. have their own innovation promotion and rewarding programmes, and federal employees who feel that creativity and innovativeness are rewarded in their organisation are more encouraged to innovate (Fernandez \& Pitts 2011). As a contribution for practitioners, we discuss the implications of our results for GOCs as well as other companies who are planning or are already running an IHCS project.

\section{Employee motivation}

Employees' motivation to be part of an innovation process is affected by multiple factors. The ability of an employee to make decisions and feel empowered is a significant factor in his or her motivation to participate in innovation processes. Although the final decision-making is vested with the top management, the impression of having an impact on the process itself supports employees' motivation to innovate. Moreover, employees are more likely to participate in innovation processes if they perceive the management's support positively (Fernandez \& Pitts 2011). It has further been discovered that communication is a crucial element with regard to employees' motivation to innovate (Gobble 2012). In the context of China, HRM practices including hiring and selection, reward, job design, and teamwork were found to impact employees' motivation and ability to be creative, but, simultaneously, neither performance appraisal nor training had any significant impact on employee creativity (Jiang et al. 2012). In another study, it was found that encouragement and support from the company's side are key factors for employees to feel bound to innovate, as also social determinants of innovation processes matter to employees (Szczepańska-Woszczyna 2014). Thus, proper human resource management can enhance trust among employees involved in innovation processes (Allen et al. 2015). 
People differ in the levels of motivation they demonstrate toward a task and in the way they are motivated (Ryan \& Deci 2000). In 1985, Deci and Ryan created the Self-Determination Theory (SDT) in which they distinguished two types of motivations, namely, internal and external, and these two types differ in terms of the motives that give rise to an action (Ryan \& Deci 2000, p.55). In intrinsic motivation, the source of inducement is from within a person, whereas extrinsic motivation is induced by external sources (Frey \& Jegen 2001). Although these types of motivation originate from different sources, as discussed below, they are not mutually exclusive (Rosenblatt 2011).

Intrinsic motivation comprises the satisfaction, curiosity, and enjoyment originating within a person toward an action and for bettering one's competence in performing said action (Frey \& Jegen 2001; Bruno 2013). Moreover, Amabile (1993, p. 188) supported this idea by stating that "individuals are intrinsically motivated when they seek enjoyment, interest, satisfaction of curiosity, self-expression, or personal challenge in the work." In brief, a person's actions are not defined or directed by external or monetary incentives but by a stance that extends beyond those incentives (Becchetti et al. 2013). In order for a person to be highly intrinsically motivated, their needs of independence and capability need to be fulfilled (Ryan \& Deci 2000). Employees tend to recognise intrinsic rewards as important, and according to Deci (1973), two factors influence intrinsic rewards. The first is the planning of job chores that employees find interesting and those that require creativity and ingenuity. The second is employees' ability to make decisions regarding their job and feeling a sense of power. For a person to be intrinsically motivated, they need to feel independent to make their own decisions, in addition to being competent enough to complete the required tasks (Ryan \& Deci 2000).

Although intrinsic motivation has been studied and proven to be a significant aspect in employees' work performance (Deci 1973), it remains somewhat of a mystery to managers. Intrinsic motivation has been disregarded in economic activities, mainly because it has been hard to pinpoint the elements in a person's job motivation that originate from intrinsic motivation (Frey \& Jegen 2001; Antoni 2009).

The general economic assumption lies in the idea that the higher the monetary compensation one receives, the better would be their work performance (Gneezy \& Rustichini 2000). Moreover, it is theoretically assumed that external incentives lead to a higher level of effort, which consequently leads to higher performance (Bonner \& Sprinkle 2002).

Extrinsic motivation in which the inducements come from an external source, such as receiving monetary compensation or reward, or avoiding punishment (Frey \& Jegen 2001; Bruno 2013), is oftentimes considered an inferior type of motivation, but it is also argued sometimes to be equal to intrinsic motivation (Kreps 1997). Individuals are considered to be extrinsically motivated when they have an external goal not related to the work itself (Ryan \& Deci 2000; Amabile 1993).

Extrinsic motivation can be divided into sub-categories depending on the amount of autonomy in decision making. In other words, extrinsic motivation can occur in situations where an individual is externally motivated to, for example, complete a task owing to outside control and fear of punishment, as well as in situations where an 
individual makes a decision based on extrinsic motivation vested in free choice and personal approval (Ryan \& Deci 2000).

As established above, the elements that build employee motivation are intrinsic and extrinsic motivation. Intrinsic motivation factors should be in balance with extrinsic motivation factors, but this balance is difficult to achieve. As a matter of fact, companies are advised to focus more on factors that promote intrinsic motivation than those that promote extrinsic motivation to obtain the best results from their employees (Deci 1973). On the one hand, employees who feel intrinsically motivated are stimulated to work by the sheer enjoyment and satisfaction derived from performing a task, as well as from the intrinsic rewards of being able to plan their own work and execute it independently. On the other hand, employees who are extrinsically motivated act upon a task because they either expect to receive compensation from an external source for completing the task or they aim to avoid making mistakes in completing the task and are thus motivated to act due to external control. Both types of motivation have been argued to be effective and to improve work performance in their own right. Although intrinsic motivation has been argued to be superior to extrinsic motivation when it comes to employee motivation, in this chapter, we evidenced the importance of extrinsic motivation in relation to work performance. Therefore, by balancing the two types of motivation suitably, maximum work performance can be expected.

\section{Participation in crowdsourcing}

When placing motivation in the context of crowdsourcing, in general, one finds that some people are motivated to participate in crowdsourcing projects because they get to share information with others (Bonabeau 2009), whereas others are motivated to participate in crowdsourcing projects or sites owing to the sheer possibility of learning something new (Boudreau \& Lakhani 2013). The possibilities of working independently and participating voluntarily increase people's motivation to participate in crowdsourcing projects (Schenk \& Guittard 2009). Although the motives for participating in a crowdsourcing project may be versatile, the initiating company should be able to identify them and recognise how they should be used to maximise the crowds' dedicated involvement in a given project (Schweitzer et al. 2012). The same can thought to be applicable to companies exercising IHCS. Without recognising the factors that motivate their crowd workers, that is, employees, an organisation might lose many potential IHCS users and their ideas.

Simula and Vuori (2012) found some challenges with internal crowdsourcing and in the way companies may motivate and engage their employees to participate in the process. For example, people need to understand the context of the idea and the framework in which the idea is executed, people do not wish to share their ideas with others, or do not think that their ideas are good enough. Moreover, one of their case companies identified the need for early adopters who would then make the others join in as well.

\section{Intrinsic and extrinsic motivation of employees in innovation processes}

Ederer and Manso (2013) found that innovation processes are better nurtured through incentive plans that accept early-stage failure and reward long-term success than 
through regular salary and pay-for-performance types of external rewards. Wendelken, Danzinger, Rau, and Moeslein (2014) found that employees who participate in organisational innovation are extrinsically motivated in terms of "career and reputation, learning, as well as firm- and peer-related issues," whereas these employees are intrinsically motivated in categories of "community, firm, fun, and enjoyment, as well as object- and task-related issues" in addition to "issues of individual connectivity and general personal attitude toward work." Furthermore, Wendelken et al. found, in accordance with existing literature, that participating employees did not feel motivated by monetary rewards, altruism, or issues related to personal needs. By contrast, non-participants in innovation projects were found to be more concerned about the lack of monetary compensation, probable stress caused by participating, and the object of the innovation project being unclear to them. Furthermore, they were found to be more distant from the organisation than the participating employees. Non-participants were also found to be most likely to be less intrinsically motivated than participants, and organisational setting and atmosphere were factors affecting their participation in innovation processes.

In the context of federal agencies, Fernandez and Pitts (2011) found out that the feeling of appreciation in the work process increases employees' willingness to innovate. The more the employees in the study felt like a part of the decision-making process regarding their work, the more willing they were to innovate. Moreover, the employees who trust and have a good relationship with their supervisor are more likely to innovate because they feel confident of being rewarded or in case of failure, not being punished. Relationships matter in the building of an atmosphere in which an employee feels encouraged to innovate; in order to get innovation from public enterprises, managers must invest in employee training and development.

It has been further concluded that employees feel increased pressure to innovate when a company is struggling and, simultaneously, increased willingness to maintain the company’s competiveness in its marketplace (Nečadová \& Scholleová 2011). This could be related to employees being afraid of an external punishment, for example, losing their job, which, in turn, increases their motivation to innovate. However, it is possible that the employees are simply genuinely concerned about the future of their employer and thus wish to assist the company by innovating, therefore being intrinsically motivated. Both motivation types, intrinsic and extrinsic, have been argued to be effective and to improve work performance.

Intrinsic motivation is generally positively linked with employees participating in innovation processes (Rosenblatt 2011), but it has also been found that extrinsic motivation through rewards and recognition does not necessarily undermine employees' willingness to innovate. Some employees might find encouragement from external rewards, whereas others might find such rewards demotivating (Rosenblatt 2011). Innovation may be found to present a change and a threat to the existing balance of an organisation and, thus, employees may not always perceive innovation as positive or embrace novel aspects that are to be implemented in an organisation (Szczepańska-Woszczyna 2014). 


\section{Methods}

\section{Research approach}

This research is based on the single-case study approach (Siggelkow 2007). The idea of the single-case study approach is to present a detailed study of one case from which a researcher may find novel relationships and question the relevance of old ones (Dyer \& Wilkins 1991). Particularly, a single-case study aims to "explore and understand how the chosen case works as a configurative and ideographic unit of analysis" (Eriksson \& Kovalainen 2008, p.121). In the present study, we employed this approach to study IHCS and employee motivation within one firm and generate suggestions based on our research results, which may be useful for other organisations as well.

\section{Case site}

The VR Group is a 150-year-old Finnish government-owned company operating mainly in the railway sector, in addition to providing bus transport and catering services. The company employs around 10,000 people and in 2014, its turnover was 1.4 billion euros. Currently, the VR Group has exclusive rights for long-distance passenger services in Finland. Recently, the VR Group's exclusivity on long-distance passenger services was extended until the end of 2024, when it is expected to be revisited. In addition to Finland, its main country of operation, the VR Group operates in Russia and Sweden.

In 2009, the VR Group began a restructuring program with the main aims of responding to the changing market environment and improving customer satisfaction by answering customer needs. One system within the restructuring program is the “Ideat kehiin” IHCS site, which was launched in 2011. It is aimed at becoming a pivotal, integrated part of the company culture in the future. This IHCS site is the representative case of IHCS in the current study. The idea behind the IHCS site is inclusion of employees in the development of the company and their own work tasks, as well as for steering the company's working environment and culture toward greater openness and inclusion. In the initial stage, the site aimed to increase the number of ideas pitched, develop good ideas that can lead to organisational development, and support the concept of pitching of ideas via an information site. At the end of stage one, a third of the company employees had participated via the site in some way. The use of IHCS in this form is based on the manner in which the platform is built and the simplicity of idea pitching. Currently, the IHCS site is in stage two, and the main intentions are to further increase the number of ideas and, especially, develop ideas that can create financial and performance benefits for the entire group. In the final stage three (2017 onwards), the site is expected to be part of the company culture and idea pitching would occur on a daily basis.

The process begins with an employee having an improvement idea on any aspect of the organisation. The employee can post the idea directly on to the IHCS site, send it as an SMS message, leave a voice mail, or send the idea in an email. The idea is then registered to the pitcher's IHCS account and posted anonymously on the IHCS site, where it can be accessed for eight weeks by others for viewing and further development. If the idea has potential, it is grouped with the 20 per cent of ideas that are passed to the decision request stage. If it passes this stage, it is approved, and the 
pitcher's name is published. Finally, the top $12 \%$ of all ideas are implemented and absorbed as new practices of the VR Group.

Currently, around a third of the company's employees have participated in the IHCS process via the site, with customer service personnel being the most active idea pitchers. Of the over 5000 ideas pitched, 260 have passed all criteria and been developed further to improve work community- and security-related issues. In addition to idea pitching, employees can comment on and "like" their colleagues" ideas on the platform. Comments are generally aimed at refining ideas, and "likes" indicate the popularity of an idea among employees.

One major aspect of the IHCS site is rewarding pitched ideas. Users are awarded points for every pitched idea, accepted idea, awarded idea, and comment. These points are convertible into euros and can be used to buy "idea prizes" in the same manner as, for example, frequent flyer miles can be used to buy products or converted directly into money. All pitchers of innovative ideas are also invited to the "Best Idea of the Year," an event in which the top ideas pitched during the year are awarded, and the pitcher of the best idea of the year is awarded 1000 euros. Other ways of encouraging people to join the site include, for example, a challenge task in which employees are to find a solution to a predefined problem.

\section{Data collection}

In the present study, data was collected via semi-structured interviews of both employees and supervisors. The interviewees were selected in close collaboration with the case company's representatives. All interviews were conducted over a period of three weeks in February 2014. We gathered people who would suit the preferred interviewee profiles, that is, subordinates who had been active in IHCS and those who had not been. In total, we conducted 18 interviews: five with supervisors and 13 with employees. In addition to primary data, we collected organisational documentation in the form of annual reports, press releases, and background information on the IHCS site because such documents provide an alternative perspective on employees' interpretations of the company or the current project.

None of the interviewed supervisors had submitted ideas of their own but had commented on their subordinates' ideas on the site. Of the 13 subordinates/employees, six had never participated on the IHCS site (created ideas or commented on ideas), whereas seven had participated. A few interviewees clearly stated that they had been told by their supervisors to attend the interview and one employee even suggested that someone else would attend the interview in his stead because, in his opinion, he did not have sufficient knowledge about the theme at hand. Despite the negative attitudes of a few participants toward the interviews, in the end, all employees were relatively active and participative in the interviews.

Of the 13 interviewed subordinates, only one was female. Similarly, of the five interviewed supervisors, one was female. Although age was not specifically asked in the interviews, the interviewees' ages ranged from 25 to 60 years, with the majority being between 35 and 45 years old. The job descriptions differed in that some were involved in customer service, while others were involved in maintenance and train driving. 


\section{Data analysis}

Ghauri (2004) suggested a two-stage analysis method for case studies. In the first stage, a general narrative of the events that occurred during data collection (e.g., in interviews and other documentation) is written, and in the second stage, the narrated data are rearranged into conceptual categories. We commenced data analysis rather subconsciously during the process of transcribing the interviews and continued in a more organised and detailed manner after all interviews were transcribed. This enabled instant interpretation of the data at hand and allowed the researchers to pose new and clarifying questions to the remaining interviewees (cf. Belk, Fischer, \& Kozinets 2013). The transcribed interviews and other documentation were then read carefully for the first time, and a few initial comments were noted on the side. During the second read-through, clear themes began to emerge and they were written down and analysed. Each interview was analysed separately so that the researchers could comprehensively understand all the different issues raised and reflect them on each other as well as on the secondary data sources.

The primary method of analysis employed in this study was thematisation. It is essential to find the essential themes from a sea of information to analyse their significance for the study. Using thematisation, one can recognise important themes in relation to the research problem and compare them with previous theoretical backgrounds. Thematisation works best when theory and empirical data are used together.

\section{Empirical findings}

The most noteworthy factors affecting employees' motivation to partake in workplace innovation via IHCS in the case company are working environment, rewards system, processing time, implementation and feedback, user experience, role of technology, and site marketing and communication. To an extent, these factors are in line those mentioned in the relevant literature, and they have been found to affect employees' motivation to innovate. In the table below, the factors are classified according to their effects on intrinsic and extrinsic employee motivation.

\begin{tabular}{|c|c|c|}
\hline & Intrinsic & Extrinsic \\
\hline Working environment & $\begin{array}{ll}\text { - } & \text { individual's feeling at work } \\
\text { - } & \text { positive, secure, and supportive }\end{array}$ & $\begin{array}{ll}- & \text { relationship with } \\
& \text { colleagues and } \\
\text { supervisors }\end{array}$ \\
\hline Rewards systems & $\begin{array}{ll}\text { - } & \text { usefulness } \\
\text { - } & \text { feeling of accomplishment }\end{array}$ & $\begin{array}{ll}\text { - } & \text { money } \\
\text { - } & \text { gratitude }\end{array}$ \\
\hline $\begin{array}{l}\text { Feedback and } \\
\text { processing time }\end{array}$ & $\begin{array}{ll}- & \text { reassurance } \\
\text { - } & \text { confidence boost }\end{array}$ & $\begin{array}{ll}\text { - } & \text { supervisor's role } \\
\text { - } & \text { time constraints }\end{array}$ \\
\hline $\begin{array}{l}\text { User experience and } \\
\text { role of technology }\end{array}$ & - $\quad$ initial success & - $\quad$ technology tools \\
\hline $\begin{array}{l}\text { Site marketing and } \\
\text { communication }\end{array}$ & $\begin{array}{ll}- & \text { goal of site } \\
- & \text { insecurity factors }\end{array}$ & - $\quad$ results of site \\
\hline
\end{tabular}

Table 3: Factors affecting employee motivation to participate in workplace innovation through IHCS

In the following chapters, the aforementioned factors are examined individually. 


\section{Working environment}

Although the role of the working environment was not particularly emphasised in the interview questions, it appeared to be a significant factor for many of the interviewees. The theme clearly stood out, and it was the first to be discussed. The theme embraces some important background information on the workers and supervisors, and their attitudes toward IHCS, in addition to raising questions pertaining to the impact of work colleagues and the current changes in the company's environment.

The VR Group is known as a traditional company with hierarchical company characteristics embedded in its culture owing to a long history of being a government bureau and then having been turned into a limited company. Since the company started the IHCS site in 2011, it has clearly been aiming not only to reinvent its innovation systems but also to reinvent the manner in which employees' wellbeing is supported. Because the VR group is government-owned, it is often perceived as a faceless corporation that has no real owner in overall charge of the company and to whom employees would feel comfortable reporting to. According to the information retrieved from the interviews, an IHCS-type of reform is welcome, although it requires some heavy reflection on the working environment, the current changes in the company's environment, and supervisor-colleague interaction.

After the 2009 change program, many organisational aspects were altered within the VR Group. Employees are still confused as to what is happening in the company and whom they should call or write an email to. This overall confusion, at least in some parts of the company, contributes to people not participating on the IHCS site. It was mentioned in the interviews that "everyday there is something new, new cell phone number, etc. It's just mostly been putting out fires, haven't had the time to get into the core yet."

One of the main issues dissuading employees from participating on the IHCS site is the lack of faith in their ability to make any actual difference. Oftentimes, the answer was "why should I do anything, it's not going to have an impact anyway" and "whatever." This shows how employees are unsure about their actual impact possibilities, most likely based on old perceptions, bad experiences, and rumors. In some cases, employee non-involvement was attributed to the general unpleasant atmosphere in the company and the company's current HR policy. In these cases, employee non-involvement actually had little to do with the IHCS site itself and more with the work environment and relationships across different organisational levels within the company.

In relation to the working environment and people's attitudes towards the IHCS site, the backgrounds and personal attributes of the workers should be taken into closer consideration. People who were clearly supportive of the site and had been active in ideation appeared to be more curious, self-imposed, and willing to help others participate via the site. On the contrary, people who felt disappointed, frustrated, and disregarded by the company, and uncertain about the site were much less inclined to participate in the IHCS process.

When asked about the roles of colleagues' and the work community's perceptions on interviewees' participation on the IHCS site, a minority of interviewees agreed that they played, mostly a negative, role in influencing the interviewees' perceptions of the site. The rest mentioned that colleagues do not really have any kind of impact on 
their participation but that it may be dispiriting to hear negatively toned rumors about the IHCS site. Such rumours might also steer a person's perception toward the negative direction. In addition, a few interviews showed that employees do not discuss IHCS with colleagues or, in general, do not have a lot to do with their colleagues.

In contrast to the above findings, in one case, it was pointed out that the main reason for employee non-participation in IHCS was possible mockery by co-workers. The IHCS site was created to be an anonymous platform, but sometimes, it is rather easy to figure out who posted an idea. Work colleagues' perception of the IHCS site appears to alter employees' perception of the site, but it does not automatically prevent the employees from using the site.

Another aspect that influences the work environment is the supervisor-subordinate relationship. One of the interview findings was that employees rarely felt the support of their supervisors and rarely felt the need to have it. When asked about what supervisors had done to encourage employees to participate on the site, the main response was "nothing." This finding might contradict the aim of the IHCS site, according to which supervisors are expected to play a leading role in commenting on ideas. In one of the major renewals of the change program in 2009, new supervisors were hired to give employees more support and the feeling that they are being listened to. However, because some of the new supervisors lacked field experience, they faced challenges in commenting on their subordinates' ideas regarding IHCS, especially on the technical aspects. This finding is unfortunate because the idea of the IHCS site was that the supervisors would, with their expertise, be the first to comment on the ideas of their subordinates.

Despite the understanding of employee motivation in IHCS in theory, the reality still seems to be far from functional because the overall impression in the employee interviews was that supervisors played a small role motivating or encouraging workers to participate on the site. Moreover, the majority of interviewees said that their supervisors had not motivated them in any significant way and do not have to do that either. From the general tone of the interviews, it was apparent that the employees did not expect their supervisors to motivate them in relation to the IHCS site. It was mentioned that a supervisor could simply not motivate a subordinate if the subordinate did not have an idea to pitch. Furthermore, if a supervisor was aware of a subordinate's active involvement in IHCS, they did not need to motivate them in any particular manner. The role of supervisors was found to be rather small in motivating employees in relation to IHCS, but their presence and relationship with their subordinates clearly impact the subordinates' participation on the site. As a matter of fact, sometimes, subordinates did not pitch in an idea because of a bad relationship with their supervisor, but once the supervisor had left, they started to submit ideas again. According to the subordinates, the main method of supervisor motivation in relation to IHCS was to share informative flyers about the site. The above issues all relate to the general working environment in the company, which affects employees' motivation to participate on the IHCS site.

Proposition 1a. Working environment increases employees' intrinsic motivation to participate in workplace innovation via IHCS when employees feel appreciated in that environment and the environment is perceived as positive, secure, and supportive. 
Proposition $1 b$. Working environment increases employees' extrinsic motivation to participate in workplace innovation via IHCS when their relationships with supervisors and colleagues are perceived as positive, secure, and supportive.

\section{Rewards system}

The rewards system of the IHCS site was found to affect employee motivation to participate on the IHCS site. In relation to the rewards system, we analyse the differences between the interviewees' motivation types.

Oftentimes, the main reason for the interviewees' non-participation on the site was "the lack of proper rewards." The points received from the creation of an idea were not the main motivation of any of the 13 employees interviewed. The rewards system was also subject to criticism, as some interviewees compared it to the rewards system that was in place prior to the current one. It appeared that the main problems employees had with the current rewards system were related to points. They were referred to as "mockery" and that receiving points felt like doing something for free. These comments came from non-participating employees. Most employees who had participated on the site perceived the points in a more positive light, but this may also be due to their intrinsic motivation toward the site. In other words, points do not play a large role from the viewpoint of intrinsically motivated employees because they would be generating ideas anyway.

However, since even the previous employee initiative system offered financial compensation for ideas, it is unlikely to consider not giving any monetary compensation for the ideas submitted to the IHCS site. Giving no reward would most probably not work, not only based on past experience but also due to the employee culture, which, as pointed out by the supervisor side, is very much run by financial incentives. An example of the influence and significance of concrete monetary compensation identified by the company's employees is that even though the old system was considered stiff and slow, many interviewees still think fondly of it because the prize for an idea was money. These interviewees are extrinsically motivated individuals, whereas intrinsically motivated interviewees stated that the old system was nothing but stiff and slow. The difference in motivation type clearly makes a difference in this case.

The rewards system divided the interviewees into the following two groups: those who felt either motivated or not demotivated by it and those who did not feel motivated by the rewards system at all. As a matter of fact, one finding in this area was that employees who were already intrinsically motivated did not feel that the points received from the IHCS site motivated them, and the non-participant employees felt more demotivated than motivated by the points system. In some cases, the points were actually the main factor keeping the non-participants from participating on the site. Both IHCS participants and non-participants felt that the company is currently receiving a disproportionate advantage in comparison to what an employee receives from pitching in an idea.

For people intrinsically motivated, the desire to do something arises from within and without external expectations. It could be said that all interviewees who participated on the IHCS site were more intrinsically motivated than extrinsically. This was evident as they expressed their opinions about the external compensation, that is, 
points: "they don't play any role," "they are not that significant," "points are as good as nothing," and "one euro here and there."

Employees who reacted negatively or indifferently to the IHCS site were found to be either extrinsically motivated or amotivated. Hence, an individual's own perception of the workability and credibility of the site seems to affect the level of intrinsic motivation. The aspect that warranted attention was that an initially intrinsically motivated employee mentioned "points are as good as nothing, you would have to do 100 ideas just to get something," where "something” means money. Intrinsically motivated people mainly said that their activity on the site would not change even if there were no monetary compensation. However, there was some variance in the answers, for example, some ideas were not pitched or commented on if no monetary reward was involved.

Interestingly, it seemed that the opportunity to win 20.000 euros increased the motivation of those employees who were otherwise considered as being driven by intrinsic motivation. Upon analysing the answers, one cannot help but wonder whether the currently intrinsically motivated employees would be actually more extrinsically motivated if they perceived the provided compensation to be desirable? This aspect seems to support previous studies in the same area in which initially performance suffered due to the introduction of compensation, but once an increase was proposed to the existing pay, performance was expected to improve again.

Proposition 2a. The rewards system increases employees' intrinsic motivation to participate in workplace innovation via IHCS when the rewards system is perceived to create a feeling of accomplishment and a sense of usefulness.

Proposition 2b. The rewards system increases employees' extrinsic motivation to participate in workplace innovation via IHCS when the rewards system involves desirable levels of monetary rewards and expression of gratitude.

\section{Feedback and processing time}

One of the biggest concerns that came up in every interview was the long processing and implementation times of ideas. It was clearly a large factor for all interviewees and one of the most criticised aspects of the entire IHCS site. Thus, unsurprisingly, a site that bases its value on the feedback channel among supervisors, their subordinates, and experts cannot be motivating if this channel is not perceived to function properly. Additionally, the old government agency culture lingers in the company and continues to affect the manner in which feedback is provided.

This aspect of the site was one of the most demotivating because the majority of the interviewees seemed to have lost faith in the processing and implementation of their ideas. It was mentioned that an idea had been pitched in the site six months prior to the interviews and had not been processed yet. Similarly, an employee's idea had taken so long to pass through all the stages that the company had implemented the idea without the person getting any recognition. The common response to the idea processing time was negative, and interviewees even appeared to have lost their trust in the site. In most interviews, the lack of quick and helpful feedback on ideas was 
brought up. An example of the disappointment toward the feedback time was the feeling of not receiving adequate appreciation or thanks for idea creation. Furthermore, the interviewees mentioned they were not properly informed as to why an idea was rejected (i.e., did not receive feedback). It was mentioned that one of the most demotivating factor is when ideas are archived without any proper explanation as to why they were not processed further. In addition, the majority of the interviewees mentioned that they have other ways of getting their ideas and opinions heard than the IHCS site and that these ways (e.g., direct contact with supervisor or an expert) are often faster and more efficient than the site.

In theory, the IHCS site comprises elements of gamification, but in practice gamification was not found to be an aspect that enhances employee motivation to participate in IHCS. This is most likely due to the long time it takes for employees to receive feedback and see their ideas implemented in practice. In fact, a small minority of the interviewees, representing Generation Y, identified IHCS as having game-like characteristics, but the other interviewees did not. The connection between receiving points by participating was insufficient to compensate for the demotivation caused by long process times. As a result, the process of providing feedback to employees suffered and was found to be inefficient. It would be ideal if the ideas could be commented on within days from their submission because "when nothing is moving onward, motivation is destroyed." Thus, the main finding is that employees wish to see immediate results and receive feedback when participating in IHCS. Currently, this is not taking place as anticipated and employees are left feeling demotivated.

Proposition 3a. Feedback increases employees' intrinsic motivation to participate in workplace innovation via IHCS when employees perceive feedback as relevant and reassuring.

Proposition 3b. Feedback and processing times increase employees' extrinsic motivation to participate in workplace innovation via IHCS when supervisors' role is perceived as positive and time constraints are minimised.

\section{User experience and role of technology}

User experience emerged as one of the themes affecting employees' motivation to participate on the IHCS site. There was a clear division between the interviewees who had positive user experiences and those who had not. Negative user experiences mainly included not getting feedback and waiting for the idea to be moved forward within the site. During the interviews, on the one hand, it was indicated that when employees did not receive any feedback on their ideas, they were unlikely for to even consider pitching an idea on the site again.

On the other hand, it was discovered during the interviews that positive user experience increased the likelihood of future activity in IHCS. This highlights the importance of positive user experiences as they can even lead, at least in the case of intrinsically motivated employees, to the development of ideas in free time. The importance of positive user experience appears to be indisputable in the case of IHCS. However, if a person is intrinsically motivated, he/she may be willing to try the site 
more times than a person who participates only for the sake of financial compensation.

In relation to user experience, technology has been found to play a significant role in IHCS. It was also found to be true in the IHCS of the case company. The interviewees responded rather positively to the large role of technology in IHCS but mentioned that occasionally challenges emerge because people do not know how to navigate on the site or have not created user IDs yet.

Technology played a negative role for a small minority, and it was the biggest reason for this minority having not participated on the site, arguing "I am not good with computers" and "ideas should be developed personally with supervisors and experts, not be left there for others to see." It was pointed out that the technology used for the IHCS platform poses a potential security risk because all company employees can access it independent of their location.

Another user experience and technology-related aspect has to do with the use of social media on the IHCS site. Two interviewees said that IHCS is a good channel for people to exchange feedback and improve the atmosphere in the working community. However, although it is positive that people aim to improve their working environment, it was also evident in some of the interviews that the employees do not appreciate the social aspect of the site. Some mentioned the social media aspect as the main reason for their non-participation.

One characteristic related to both user experience and technology that came up during various interviews was speculated misuse of the site. Site misuse manifests in the form of pointless comments or simple ideas with no actual development value to the company. Because every idea earns a person three points (three euros) and each comment one point (one euro), it was speculated whether some users have simply begun collecting money from the IHCS site and, consequently, eroding its credibility. It was mentioned that the line between the ideas that should be written on the site and the ones that should not had blurred and that in some cases, the ideas had "gotten out of hand." The speculated misuse of the site had not affected interviewees' participation to a great extent, but many of them are aware of it and clearly annoyed by it. The misuse of the site may diminish its credibility in the eyes of current and future users and should thus be contained in some way. Although misuse seems evident, it is impossible to say whether some commenters simply find some ideas worthy of multiple comments and are not intentionally misusing the site for points. In relation to the social media aspect, the types of ideas that should be submitted on the site were discussed. A small minority of the interviewees found ideas and comments about the social aspect of work to be acceptable, whereas others thought that the submitted ideas should be strictly innovative and aim to develop the company

Proposition 4a. User experience increases employees' intrinsic motivation to participate in workplace innovation via IHCS when the initial user experience is perceived as successful and positive.

Proposition 4b. Technology increases employees' extrinsic motivation to participate in workplace innovation via IHCS when it is perceived as easy to use and helpful. 


\section{Site marketing and communication}

During the interviews, it became apparent that some of the interviewees were not completely aware of the workings of the IHCS site. When asked about the advantages, internal or external, of the site to the VR Group, the majority mentioned that they had no idea. From the subordinate interviews, it could be said that for whatever reason, there is a communications blockage across different levels of the company. When basic information about the site does not reach everyone, false misconceptions may arise, damaging the image of the site. The company has recognised that communication and interaction regarding changes need to be developed further. Although communication remains an issue, the company is aiming to improve it. During the interviews, it was suggested that in order to motivate employees to participate on the site, the company should communicate some concrete results created by the site. In other words, the company should show what has been achieved by implementing an employee's idea, it costs, and its eventual benefit in terms of money saved by the company. Furthermore, more internal marketing was demanded by the non-participants, who stated that while it would be motivational to see the concrete results of an idea, the focus should be on the development process and not the person who performing the process. Despite the demand for more internal marketing, some objections were made against it owing to the fear that with increased solicitation of idea initiators, the number of individuals attempting to misuse the site would increase.

In relation to communication and internal marketing of the IHCS site, not all of the interviewees were familiar with the site's goal. Some actually admitted to not being aware of the goal and the reason for having an IHCS site. According to them, there was a lack of marketing, in addition to personal inactivity on the site. Many could not say in which ways the goals motivated them, whereas others were certain that the goals do not motivate them to participate in any way. The interviewees who expressed an understanding of the site's goals listed them to be improvement of company performance and working environment, down-to-top innovation, cost savings, and service development.

Proposition 5a. Site marketing and communication increase employees' intrinsic motivation to participate in workplace innovation via IHCS when marketing and communication articulate the goal and purpose of the site while simultaneously alleviating security fears.

Proposition 5b. Site marketing and communication increases employees' extrinsic motivation to participate in workplace innovation via IHCS when marketing and communication present positive tangible results of IHCS. 


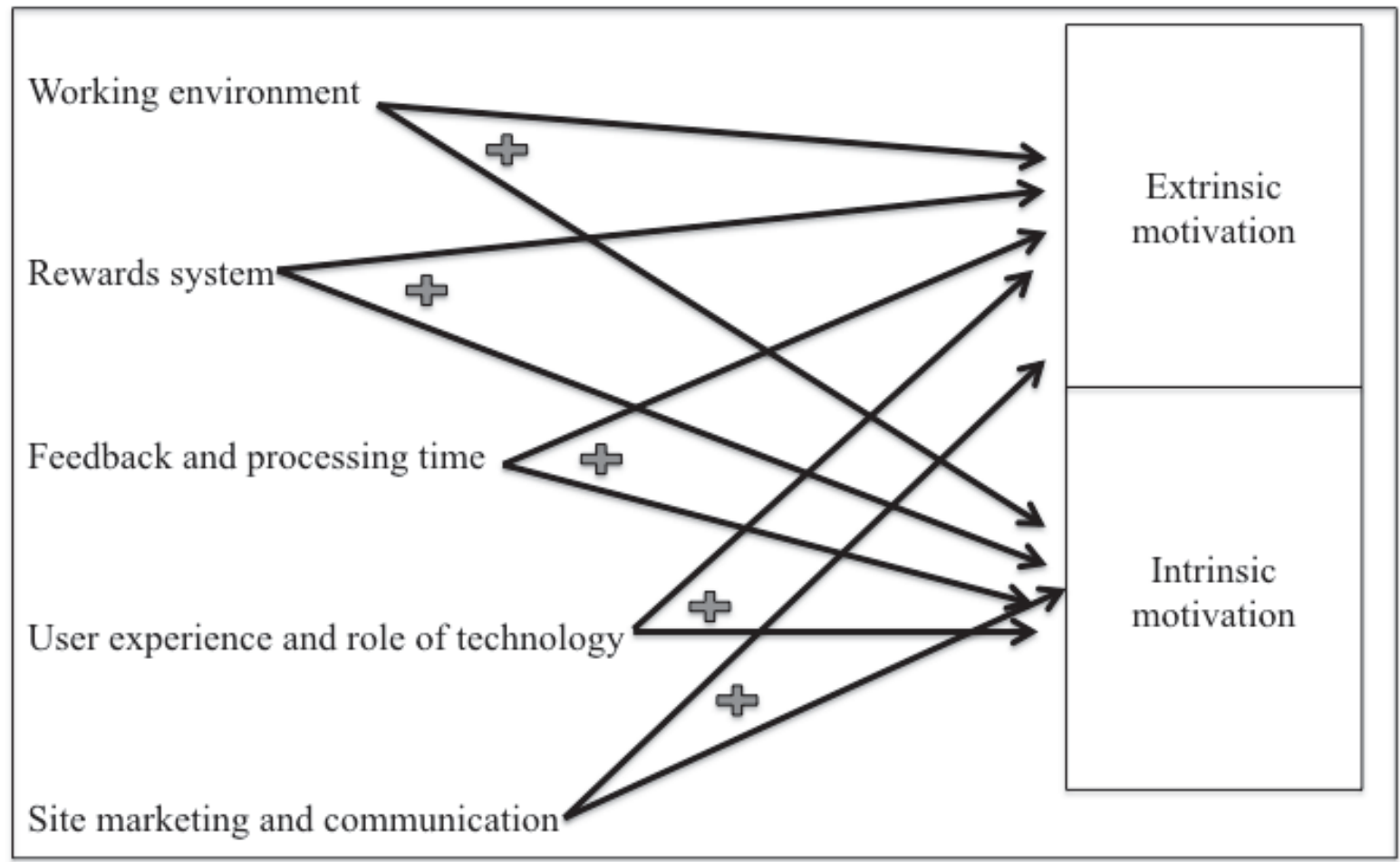

Figure 2 Factors affecting employee motivation to participate in workplace innovation via IHCS

As presented in Figure 1, all five factors affecting employee motivation to participate in workplace innovation via IHCS affect both intrinsic and extrinsic motivation. The five motivation factors, working environment, rewards system, feedback and processing time, user experience and role of technology, and site marketing and communication are found to positively affect both intrinsic and extrinsic motivations of employees. In the following section, we present conclusions made based on these findings and list both managerial and theoretical implications.

\section{Conclusions}

Although the benefits of workplace innovation (Black \& Lynch 2004; Pot 2011) are encouraging, the research focusing on employee motivation to participate in workplace innovation practices is still in its infancy. In this study, we aimed to identify the factors that affect employee motivation to participate in workplace innovation via IHCS, namely working environment, rewards system, feedback and processing time, user experience and role of technology, and site marketing and communication. 
The results of this study suggest that in the context of workplace innovation, motivation toward action originates from both intrinsic and extrinsic sources. This finding is somewhat in contradiction to the findings of previous studies, which found employee participation to exemplify unassuming readiness to contribute to organisational accomplishment (Albrecht 2010, p.5). This study argues that although intrinsic motivation is important for participation in workplace innovation, extrinsic rewards are required to motivate extrinsically motivated individuals. A balance between extrinsic and intrinsic motivators must be created to secure employee participation in IHCS.

This research contributes to existing workplace innovation literature by presenting a unique take on employee motivation in the context of IHCS. Interestingly, the study discusses employee motivation in the context of a large GOC. We indicate some hurdles faced by a company of this specific nature and the influence of said hurdles on company culture. Furthermore, we discuss the influence of working environment, rewards system, feedback and processing time, user experience and role of technology, and site marketing and communication on employee motivation to participate in workplace innovation in general, and the IHCS process in particular.

Given that the study is limited to a single case company with defining company characteristics, generalising the results is challenging. However, in terms of further research, researchers dealing with this subject could possibly use our results to conduct comparative studies on different types of organisations for determining whether some universality exists in motivating employees to participate in workplace innovation via IHCS or whether there are significant differences between GOCs and private businesses.

\section{Managerial implications}

The results of this study are useful for organisations using IHCS to facilitate workplace innovation or for organisations planning to implement it. Based on the findings, companies already using IHCS may wish to revisit their IHCS policies and understand the factors that influence their employees in terms of participating in IHCS.

Based on the information in this study, it would be advisable for companies planning to implement IHCS to evaluate the type of rewards that appeal to their employees, namely, intrinsic or extrinsic, and implement a rewards system accordingly while bearing in mind that intrinsic motivation is oftentimes the prerequisite for long-term employee participation. Once compensation is introduced, it tends to eat up the initial intrinsic motivation and causes employees to get used to and be directed by the expectation of compensation.

\section{Limitations and suggestions for further research}

Since the case company presented in this study is a large GOC, it possesses many characteristics not found in private or small and medium-sized companies. Moreover, as the results of the study are based on one specific case company, they may not be directly generalisable to other companies. To elaborate, companies not sharing the defining characteristics of the case company may not be facing similar challenges in terms of employee motivation in workplace innovation or not at least to the same 
extent as the case company. As the results of this study focus on employee motivation in the context of a large company, they may not be applicable to smaller companies. Additionally, small and medium-sized companies do not generally possess resources to the same extent as a large company and may thus not be able to change their operations to the same degree as large companies. As public organisations tend to attract different types of people than private companies (Perry \& Porter 2012), the research results are limited solely to representing employee motivation factors in this particular environment. If a similar type of research was conducted in a private company, the results may differ from the results of this study. The empirical research is also limited to Finland and is thus bound by cultural features that are likely to differ greatly in comparison to those in other countries.

Given that the combination of the IHCS and employee motivation concepts is novel, the possibilities for further research are vast. A suggestion for further research would be to test the theoretical propositions made in this study. Moreover, it would be interesting to compare different types of companies varying in size, industry, and company culture, and research how employees in different types of organisations react to IHCS. In addition, a comparison of a private company's IHCS and a GOC's IHCS may provide new insights into their differences and suggest possibilities for knowledge integration. In case significant variance is found, one could further investigate the reasons underlying the variance and determine whether a universal framework on IHCS engagement could be drawn. An additional future research direction could focus on supervisors' attitudes and opinions on IHCS and the effects thereof on the manner in which they encourage their subordinates. 


\section{References}

Aalbers, R., Dolfsma, W. \& Koppius, O., 2013. Individual connectedness in innovation networks: On the role of individual motivation. Research Policy, 42(3), pp.624-634.

Albrecht, S.L., 2010. Handbook of Employee Engagement: Perspectives, Issues, Research and Practice, Edward Elgar: Cheltenham.

Allen, M.R., Adomdza, G.K. \& Meyer, M.H., 2015. Managing for innovation: Managerial control and employee level outcomes. Journal of Business Research, 68(2), pp.371-379.

Amabile, T.M., 1993. Motivational synergy: Toward new conceptualizations of intrinsic and extrinsic motivation in the workplace. Human Resource Management Review, 3(3), pp.185-201.

Antoni, G.D., 2009. Intrinsic vs. Extrinsic Motivations to Volunteer and Social Capital Formation. Kyklos, 62(3), pp.359-370.

Becchetti, L., Castriota, S. \& Tortia, E.C., 2013. Productivity, wages and intrinsic motivations. Small Business Economics, 41(2), pp.379-399.

Belk, R., Fischer, E. \& Kozinets, R. V., 2013. Qualitative consumer \& marketing research, London, UK: SAGE Publications.

Black, S.E. \& Lynch, L.M., 2004. What's driving the new economy?: The benefits of workplace innovation. The Economic Journal, 114(February), pp.F97-F116.

Bonabeau, E., 2009. Decisions 2.0 : The Power of Collective Intelligence. MIT Sloan Management Review, 50(2), pp.45-52.

Bonner, S.E. \& Sprinkle, G.B., 2002. The effects of monetary incentives on effort and task performance: Theories, evidence, and a framework for research. Accounting, Organizations and Society, 27(4-5), pp.303-345.

Boudreau, K.J. \& Lakhani, K.R., 2013. Using the Crowd as an Innovation Partner. Harvard Business Review, 91(4), pp.60-69.

Bruno, B., 2013. Reconciling economics and psychology on intrinsic motivation. Journal of Neuroscience, Psychology, and Economics, 6(2), pp.136-149.

Deci, E.L., 1973. Paying people doesn't always work the way you expect it to. Human Resource Management, 12(2), pp.28-32.

Dyer, W.G. \& Wilkins, A.L., 1991. Better Stories, Not Better Constructs, To Generate Better Theory: A Rejoinder To Eisenhardt. Academy of Management Review, 16(3), pp.613-619.

Ederer, F. \& Manso, G., 2013. Is Pay for Performance Detrimental to Innovation? Management Science, 59(7), pp.1496-1513.

Eriksson, P. \& Kovalainen, A., 2008. Qualitative methods in business research, London, UK: Sage Publications.

Fernandez, S. \& Pitts, D.W., 2011. Understanding Employee Motivation to Innovate: Evidence from Front Line Employees in United States Federal Agencies. Australian Journal of Public Administration, 70(2), pp.202-222. 
Frey, B. \& Jegen, R., 2001. Motivation crowding theory: a survey of empirical evidence. Journal of Economic Surveys, 15(5), pp.589-611.

Ghauri, P.N., 2004. Designing and conducting case studies in international business research. In R. Marschan-Piekkari \& C. Welch, eds. Handbook of qualitative research methods for international business. Edward Elgar: Cheltenham, pp. 109-124.

Gneezy, U. \& Rustichini, A., 2000. Pay Enough or Don’t Pay at All. The Quarterly Journal of Economics, 115(3), pp.791-810.

Gobble, 2012. Resources: Motivating Innovation. Research-Technology Management, 55(6), pp.66-68.

Howe, J., 2006. The Rise of Crowdsourcing. Wired Magazine, 14(06), pp.1-5.

Jiang, J., Wang, S. \& Zhao, S., 2012. Does HRM facilitate employee creativity and organizational innovation? A study of Chinese firms. The International Journal of Human Resource Management, 23(19), pp.4025-4047.

Kesting, P. \& Ulhøi, J.P., 2010. Employee-driven innovation: extending the license to foster innovation. Management Decision, 48(1), pp.65-84.

Kreps, D., 1997. Intrinsic motivation and extrinsic incentives. The American Economic Review, 87(2), pp.359-364.

Nečadová, M. \& Scholleová, H., 2011. Motives and Barriers of Innovation Behaviour of Companies. Economics \& Management, 16, pp.832-838. 7p. 2 Charts.

Perry, J.L. \& Porter, L.W., 2012. Factors affecting the context for motivation in public organizations. Academy of Management Review, 7(1), pp.89-98.

Pot, F., 2011. Workplace innovation for better jobs and performance. International Journal of Productivity and Performance Management, 60(4), pp.404-415.

Rosenblatt, M., 2011. The use of innovation awards in the public sector: Individual and organizational perspectives. Innovation: Management, Policy and Practice, 13(2), pp.211-223.

Ryan, R. \& Deci, E., 2000. Intrinsic and Extrinsic Motivations: Classic Definitions and New Directions. Contemporary educational psychology, 25(1), pp.54-67.

Schenk, E. \& Guittard, C., 2009. Crowdsourcing: What can be Outsourced to the Crowd, and Why?

Schweitzer, F.M. et al., 2012. Crowdsourcing: Leveraging Innovation through Online Idea Competitions. Research-Technology Management, 55(3), pp.32-38.

Siggelkow, N., 2007. Persuasion with case studies. Academy of Management Journal, 50(1), pp.20-24.

Simula, H. \& Ahola, T., 2014. A network perspective on idea and innovation crowdsourcing in industrial firms. Industrial Marketing Management, 43(3), pp.400408.

Simula, H. \& Vuori, M., 2012. Benefits and Barriers of Crowdsourcing in B2B Firms: Generating Ideas With Internal and External Crowds. International Journal of Innovation Management, 16(06), p.1240011.

Stieger, D. et al., 2012. Democratizing Strategy: How Crowdsourcing Can Be Used for Strategy Dialogues. California Management Review, 54(4), pp.44-68. 
Szczepańska-Woszczyna, K., 2014. Innovation processes in the social space of the organization. Regional Formation and Development Studies, 14(3), pp.220-229.

Totterdill, P., 2015. Closing the Gap : The Fifth Element and Workplace Innovation. European Journal of Workplace Innovation, 1(1), pp.55-74.

Wendelken, A. et al., 2014. Innovation without me: Why employees do (not) participate in organizational innovation communities. R\&D Management, 44(2), pp.217-236.

Villarroel, J.A. \& Reis, F., 2010. Intra-Corporate Crowdsourcing (ICC): Leveraging Upon Rank and Site Marginality for Innovation. In Proceedings of Crowdconf 2010: The World's First Conference on The Future of Distributed Work.

Väätänen, J. et al., 2011. New Trends in Russian Innovations: The Ownership Effect on the Adoption of Open Innovation Practices. Journal of East-West Business, 17(23), pp.132-143.

\section{About the authors:}

Kira Palin

M.Sc. in Economics and Business Administration (Global Innovation Management), Turku School of Economics. Independent researcher.

\section{Valtteri Kaartemo}

D.Sc. in Economics and Business Administration, Turku School of Economics. University Teacher. 